\title{
SEMENTES DE FEIJÃO-CAUPI (Vigna unguiculata L. (Walp), TRATADAS COM EXTRATO DE FOLHAS DE NIM (Azadirachta indica A. Juss.) AVALIAÇÃO DA GERMINAÇÃO E DA INCIDÊNCIA DE FUNGOS
}

\section{COWPEA SEEDS (Vigna unguiculata L. (Walp), TREATED WITH NIM LEAF EXTRACT (Azadirachta indica A. Juss.) AVALIATION OF GERMINATION AND FUNGI INCIDENCE}

\author{
Gilvânia Campos SILVA ${ }^{1}$ \\ Delineide Pereira GOMES ${ }^{2}$ \\ Ceália Cristine SANTOS ${ }^{3}$
}

\begin{abstract}
RESUMO
Este estudo teve o objetivo de avaliar o efeito do extrato aquoso de folhas de nim sobre a germinação e incidência de fungos em sementes de três cultivares (Serrinha, BR-17 e Maranhão) de feijão-caupi. Folhas de nim foram secas, trituradas e preparadas diluições de 0,$5 ; 1,0 ; 2,0$ e $4,0 \mathrm{~g} \mathrm{dm}^{-3}$ e testemunha. Avaliou-se a incidência de fungos, pelo teste de papel de filtro e a germinação de acordo com as Regras para Análise de Sementes. Nas três cultivares analisadas, observouse redução na incidência de Aspergillus sp e Fusarium sp. Em relação à influência dos extratos das folhas de nim sobre a germinação das sementes, observou efeito significativo do extrato na cultivar Maranhão, onde todas as concentrações diferiram da testemunha e proporcionaram aumento considerável na porcentagem de plântulas normais. Conclui- se que o extrato de folhas de nim foi efetivo no controle de Aspergillus sp, Fusarium sp, Phoma sp e Macrophomina phaseolina em diferentes concentrações nos diferentes cultivares e a germinação das sementes foi estimulada para a cultivar Maranhão.

Palavras-chave: qualidade; sanitária; fisiológica; Fusarium; Macrophomina; Aspergillus; Phoma.
\end{abstract}

\begin{abstract}
This study aimed to evaluate the effect of aqueous extract of neem on germination and fungi incidence on seeds of three cultivars (Serrinha, BR 17 and Maranhão) of cowpea. Neem leaves were dryed, crushed and prepared dilutions of 0.5 ; $1.0 ; 2.0,4.0 \mathrm{~g} \mathrm{dm}^{-3}$ and control. The fungi incidence was evaluated by the test filter paper and germination according to the Rules for Seeds Testing (Regras para Análise de Sementes). In the three cultivars analyzed, reduction in the incidence of Aspergillus sp and Fusarium sp was observed. In relation to the influence of extracts of neem leaves on seed germination, significant effect of extract in Maranhão cultivar was observed, where all concentrations differed from the control, and propovided a considerable increase in the percentage of normal seedlings. It was concluded that the leaf extract of neem was effective in controlling Aspergillus sp, Fusarium sp, Phoma sp and Macrophomina phaseolina at different concentrations in different cultivars and seed germination was stimulated for the Maranhão cultivar.

Key-words: quality; sanitary; physiological; Fusarium; Macrophomina; Aspergillus; Phoma
\end{abstract}

\footnotetext{
${ }^{1}$ Bióloga, Doutoranda em Microbiologia - FCAV/UNESP, Via de Acesso Prof. Paulo Donato Castellane, s/n, CEP. 4884-900, Jaboticabal-SP, email: gilvaniacampos@ig.com.br;

${ }^{2}$ Engenheira Agrônoma, Doutoranda em Fitotecnia, Universidade Federal de Viçosa - UFV, Viçosa, MG, Brasil. agroneide@hotmail.com .

${ }^{3}$ Geógrafa, Mestre em Agroecologia, Professora da Universidade Federal do Maranhão. cealia@ig.com.br
} 


\section{INTRODUÇÃO}

O uso de compostos químicos sintéticos tem sido, há muitos anos, um procedimento eficiente para o controle de doenças de plantas. Apesar de sua significativa contribuição para a produção agrícola, o uso intensivo e indiscriminado desses produtos contribuiu para o surgimento de pragas secundárias e não conseguiu eliminar os problemas já existentes; além disso, são altamente tóxicos, sendo prejudiciais aos aplicadores e consumidores e ao meio ambiente (Casa, 2005).

Atualmente há a tendência de serem utilizados produtos alternativos à base de recursos provenientes de plantas que podem ser cultivadas fornecendo a matéria prima (extratos ou óleos essenciais) para ser utilizada na agricultura e substituir os agroquímicos. As plantas podem apresentar em sua composição química, substâncias ativas que podem atuar nas interações planta-patógeno como fungitóxicas, outras com atividade antimicrobiana ou, ainda, ativando os mecanismos de defesa da planta hospedeira, objetivando o controle de patógenos e, paralelamente, a redução do uso indiscriminado de defensivos. A essas vantagens, acrescenta-se o fato de serem facilmente biodegradáveis, seletivas e de fácil obtenção e utilização pelos produtores, terem baixo custo e minimizarem os problemas apresentados pelos produtos químicos sintéticos.

Extratos biologicamente ativos obtidos de folhas, frutos, sementes e tronco de nim (Azadirachta indica A. Juss.) são reconhecidos por suas propriedades inseticidas, sendo a azadiractina considerada o composto ativo mais potente da árvore de nim e está presente em todas as partes da planta. O óleo de nim é, geralmente, obtido das sementes prensadas e apresentam maior concentração do composto que o extrato das folhas. Vários trabalhos têm sido realizados usando esta planta no controle de pragas e patógenos e muitos resultados positivos têm sido obtidos, comprovando a eficácia de suas propriedades.

Diante do exposto, considerando o potencial do nim para uso no controle do desenvolvimento de fungos, o objetivo do trabalho foi avaliar o efeito do extrato aquoso de nim sobre a incidência de fungos e a germinação de sementes de feijão-caupi.

\section{MATERIAL E MÉTODOS}

Folhas de nim foram secas em estufa à temperatura de $50^{\circ} \mathrm{C}$, por $24 \mathrm{~h}$, trituradas e colocadas em água destilada numa proporção de $60 \mathrm{~g} \mathrm{dm}^{-3}$ durante $24 \mathrm{~h}$. A partir dessa suspensão foram preparadas diluições em água de 0,0 (testemunha); 0,$5 ; 1,0 ; 2,0$ e $4,0 \mathrm{~g} \mathrm{dm}^{-3}$ As sementes de feijãocaupi foram submersas por $10 \mathrm{~min}$, em seguida secas em papel filtro, em temperatura ambiente. $O$ teste de sanidade foi realizado, pelo método do papel de filtro (Lucca Filho, 1987), com quatro repetições de 50 sementes. As sementes foram incubadas por sete dias a $20 \pm 2{ }^{\circ} \mathrm{C}$ e fotoperíodo de $12 \mathrm{~h}$ sob luz branca fluorescente e examinadas, individualmente, sob microscópio estereoscópico, para a detecção de fungos. Quando necessário, a análise foi complementada com a observação de lâminas ao microscópio ótico.

A germinação das sementes foi avaliada considerando as informações das Regras para Análise de Sementes (Brasil, 2009). O teste padrão de germinação foi realizado empregando-se, como substrato, areia previamente peneirada, lavada e esterilizada em autoclave. Foram utilizadas 4 repetições de 50 sementes, semeadas em areia, em caixas plásticas, e mantidas em condições ambiente de laboratório. A avaliação foi realizada sete dias após a instalação do experimento, através da contagem de plântulas normais e plântulas anormais e os resultados foram expressos em porcentagem.

Foram utilizadas sementes de três cultivares de feijão-caupi: a variedade Serrinha, proveniente da cidade de Timon-MA, Maranhão da cidade de Viana - MA e BR 17 obtida junto à Embrapa Meio Norte na cidade de Terezina - PI. O experimento foi instalado em esquema fatorial do tipo 5 (Concentrações) x (Cultivares), os dados de porcentagem foram transformados em arco seno $(x)$ 0,5. A análise de variância foi feita através do teste $F$ e a comparação de médias, através do teste de Tukey ( $5 \%$ de probabilidade).

\section{RESULTADOS E DISCUSSÃO}

Foram identificados nas sementes de feijãocaupi os seguintes fungos: Aspergillus sp, Fusarium sp, Macrophomina phaseolina e Phoma sp. Na Tabela 1 , observa-se que a cultivar BR 17 teve uma redução de $51,86 \%$ na incidência de Aspergillus sp, quando as sementes foram tratadas com extrato de folhas de nim na concentração $4,0 \mathrm{~g} \mathrm{dm}^{-3}$. Para as sementes da cultivar Maranhão ocorreu redução de 11,33 e $11,09 \%$ com 2,0 e $4,0 \mathrm{~g} \mathrm{dm}^{-3}$ do extrato e na cultivar Serrinha os resultados mostram que houve aumento na incidência de Aspergillus sp na ordem crescente das concentrações do extrato de folhas de nim. Aspergillus spp não são patogênicos à cultura do feijão- caupi, sendo considerados fungos de armazenamento. Todavia é importante evitar o desenvolvimento destes nas sementes pois, segundo Torres e Bringel (2005) podem causar danos como redução de germinação, aumento da taxa de ácidos graxos, descoloração da semente, alterações bioquímicas, produção de toxinas prejudiciais ao homem e outros animais e redução do peso seco da semente.

Utilizando extrato de nim, Ferreira et al. (2006) reduziram a incidência de Aspergillus niger associados a sementes de embiratanha (Pseudobombax grandiflorum (CAV). A. Rob.), a 48 $\mathrm{g} \mathrm{dm}^{-3}$ com $50 \mathrm{~g} \mathrm{dm}^{-3}$ do extrato e a $51 \mathrm{~g} \mathrm{dm}^{-3} \mathrm{com}$ $100 \mathrm{~g} \mathrm{dm}^{-3}$ de extrato, comparando com $78 \mathrm{~g} \mathrm{dm}^{-3}$ do fungo presente nas sementes não tratadas. Campos (2010) constatou que em sementes de algodão, o extrato da folha de nim não afetou o crescimento de Aspergillus flavus, porém bloqueou a produção de aflatoxina.

Em relação aos fungos do gênero Fusarium, constatou-se redução da incidência do fungo em todas as concentrações do produto quando comparados com a testemunha, entretanto, não 
SILVA.G.C et al. Sementes de feijão-caupi...

houve diferença entre as diferentes concentrações sementes das três cultivares (Tabela 2). avaliadas. Esse mesmo resultado foi observado nas

TABELA 1 - Incidência (\%) de Aspergillus sp. em sementes de feijão-caupi, testemunha e tratadas com extrato aquoso de folhas de $\operatorname{nim}\left(0,5 ; 1,0 ; 2,0\right.$ e $\left.4,0 \mathrm{~g} \mathrm{dm}^{-3}\right)$

\begin{tabular}{lccc}
\hline & \multicolumn{3}{c}{ Cultivares } \\
\hline $\begin{array}{l}\text { Concentração }\left(\mathrm{g} \mathrm{dm}^{-3}\right) \text { de } \\
\text { extrato de nim }\end{array}$ & BR 17 & Maranhão & Serrinha \\
& & & \\
\end{tabular}

Testemunha $(0,0)$

$70.05 a b$ A

36,44 a B

8,92 b C

0,5

68.35 a A

$18,64 a b B$

$36,70 \mathrm{aB}$

1,0

50.51 bc $A$

$18,22 \mathrm{abB}$

38,74 a $A B$

2,0

44.16 bc $A$

4,13 b B

40,20 ab B

4,0

36.33 с A

4,04 b B

42,35 a A

\begin{tabular}{llll}
\hline $\mathrm{CV}(\%)$ & 4,5 & 17,0 & 23,0
\end{tabular}

TABELA 2 - Incidência (\%) de Fusarium sp. em sementes de feijão-caupi, testemunha e tratadas com extrato aquoso de folhas de nim ( 0,$5 ; 1,0 ; 2,0$ e $\left.4,0 \mathrm{~g} \mathrm{dm}^{-3}\right)$

\begin{tabular}{cccc}
\hline & \multicolumn{3}{c}{ Cultivares } \\
\hline $\begin{array}{c}\text { Concentração }\left(\mathrm{g} \mathrm{dm}^{-3}\right) \\
\text { de extrato de nim }\end{array}$ & BR 17 & Maranhão & Serrinha \\
\hline Testemunha $(0,0)$ & 60,00 a A & 84,00 a A & 54,50 a A \\
0,5 & 46,00 b A & 40,50 b A & 38,00 b A \\
1,0 & 46,00 b A & 32,50 b A & 30,00 b A \\
2,0 & 34,00 b A & 32,50 b A & 26,50 b A \\
4,0 & 22,50 b A & 26,50 b A & 24,00 b A \\
\hline CV $(\%)$ & 12,0 & 36,0 & 15,0 \\
\hline
\end{tabular}

Médias seguidas de mesma letra maiúscula nas linhas e minúscula, nas colunas, não diferem pelo teste de Tukey ( $5 \%$ de probabilidade). C.V = Coeficiente de Variação

Silva et al. (2006) utilizaram óleo de nim, no controle de Fusarium oxysporum, e observaram que houve redução do patógeno nas concentrações de 1,88 e $2,51 \mathrm{~g} \mathrm{dm}^{-3}$. Souza et al. (2006) utilizaram diferentes concentrações de óleo de nim $(0,5 ; 0,7$; 0,$9 ; 1,1 ; 1,3 \mathrm{~g} \mathrm{dm}^{-3}$ ) e constataram que houve controle de Fusarium moniliforme a partir de $0,5 \mathrm{~g} / \mathrm{dm}^{-3}$ e Fusarium subglutinans, apenas com $1,3 \mathrm{~g} \mathrm{dm}^{-3}$. Os fungos do gênero Fusarium são importantes para a cultura do feijão-caupi, pois há espécies que são patogênicas e segundo Pinto (1999) promovem redução na germinação e tombamento de plântulas.

No que se refere à Macrophomina phaseolina, as sementes das cultivares BR 17 e Maranhão não apresentaram qualquer alteração na incidência desse patógeno. Na cultivar Serrinha, notase que houve uma redução de cerca de $68 \%$ na incidência do fungo, nas diferentes concentrações em relação à testemunha, conforme mostra a Tabela 3. Este fungo também não foi inibido, quando Pessoa et al. (2003) usaram extrato de folhas secas de Ocimum basilicum nas concentrações de 5; 10; 15 e $20 \mathrm{~g} \mathrm{dm}^{-3}$. Todavia o extrato de sementes de nim a $0,5 \mathrm{~g} \mathrm{dm}^{-3}$ e $1 \mathrm{~g} \mathrm{dm}^{-3}$ inibiu o crescimento de Alternaria alternata e Macrophomina phaseolina em trabalho realizado por Bhutta (2001).

Em relação aos fungos do gênero Phoma, (Tabela 4) somente a cultivar Maranhão apresentou redução de $24 \%$ na incidência desse fungo quando são comparadas as diferentes concentrações do extrato de folhas de nim com o tratamento testemunha, sem aplicação de produtos.

Mossini e Kemmelmeier (2005) demonstraram que os extratos de nim possuem atividade fungitóxica, apesar de seu modo de ação não ser ainda totalmente esclarecido. É possível que os diferentes compostos ou as diferentes concentrações dos compostos extraídos da planta possuam efeitos variados em fungos. Existem evidências, fornecidas por este e outros estudos, que fungos reagem diferentemente aos compostos de nim como observado por Govindachari et al. (1998), que estudando a 
SILVA,G.C et al. Sementes de feijão-caupi...

atividade antifúngica de terpenóides constituintes do óleo de nim, observaram que a azadiractina não afetou o crescimento de três fungos fitopatogênicos, enquanto que a salanina, nimbina, epoxiazadiradiona, deacetilnimbina e azadiradiona, apresentaram diferentes níveis de controle.

TABELA 3 - Incidência (\%) de Macrophomina phaseolina em sementes de feijão-caupi, testemunha e tratadas com extrato aquoso de folhas de $\operatorname{nim}\left(0,5 ; 1,0 ; 2,0\right.$ e $\left.4,0 \mathrm{~g} \mathrm{dm}^{-3}\right)$.

\begin{tabular}{cccc}
\hline & \multicolumn{2}{c}{ Cultivares } \\
\hline $\begin{array}{c}\text { Concentração }\left(\mathrm{g} \mathrm{dm}^{-3}\right) \text { de } \\
\text { extrato de nim }\end{array}$ & BR 17 & Maranhão & Serrinha \\
\hline Testemunha $(0,0)$ & 15,00 a B & 17,00 a B & 25,00 a A \\
0,5 & 15,00 a A & 16,00 a A & 17,50 b A \\
1,0 & 15,00 a A & 15,00 a A & 17,00 b A \\
2,0 & 15,00 a A & 15,00 a A & 16,00 b A \\
4,0 & 15,00 a A & 15,00 a A & 16,00 b A \\
\hline CV $(\%)$ & 0,0 & 2,0 & 7,0 \\
\hline
\end{tabular}

Médias seguidas de mesma letra maiúscula nas linhas e minúscula, nas colunas, não diferem pelo teste de Tukey $(5 \%$ de probabilidade). C.V = Coeficiente de Variação

TABELA 4 - Incidência (\%) de Phoma sp. em sementes de feijão-caupi, testemunha e tratadas com extrato aquoso de folhas de $\operatorname{nim}\left(0,5 ; 1,0 ; 2,0\right.$ e $\left.4,0 \mathrm{~g} \mathrm{dm}^{-3}\right)$

\begin{tabular}{cccc}
\hline & \multicolumn{2}{c}{ Cultivares } \\
\hline $\begin{array}{c}\text { Concentração }\left(\mathrm{g} \mathrm{dm}^{-3}\right) \text { de } \\
\text { extrato de nim }\end{array}$ & BR 17 & Maranhão & Serrinha \\
\hline $\begin{array}{c}\text { Testemunha }(0,0) \\
0,5\end{array}$ & 10,00 a B & 50,00 a A & 10,00 a B \\
1,0 & 10,00 a B & 12,50 b A & 10,00 a B \\
2,0 & 10,00 a B & 12,50 b A & 9,50 a B \\
4,0 & 10,00 a B & 12,50 b A & 9,50 a B \\
CV (\%) & 10,00 a B & 12,00 b A & 9,50 a B \\
\hline
\end{tabular}

Médias seguidas de mesma letra maiúscula nas linhas e minúscula, nas colunas, não diferem pelo teste de Tukey ( $5 \%$ de probabilidade). C.V = Coeficiente de Variação

Foi avaliada também a influência dos extratos de folhas de nim sobre a germinação das sementes e só se observou efeito significativo do extrato para as sementes da cultivar Maranhão, em que as diferentes concentrações do extrato, diferiram da testemunha e proporcionaram aumento na porcentagem de plântulas normais (Tabela 5). As cultivares responderam de maneira diferente ao ataque de fungo e também de maneira diferente à ação do extrato de folha de nim. É provável que o princípio ativo da planta estimule a germinação das sementes de algumas cultivares como ocorreu com a Maranhão e não seja eficaz em outras como a Serrinha e BR 17.

TABELA 5 - Germinação (\%) de sementes de feijão-caupi, das cultivares BR 17, Maranhão e Serrinha, tratadas com extrato de folhas de nim, em quatro concentrações

\begin{tabular}{cccc}
\hline & Cultivares & Serrinha \\
\hline $\begin{array}{c}\text { Concentração }\left(\mathrm{g} \mathrm{dm}^{-3}\right) \text { de } \\
\text { extrato de nim }\end{array}$ & BR 17 & Maranhão & 57 b A \\
\hline Testemunha $(0,0)$ & 59 b A & 36 b B & 52 b A \\
0,5 & 59 b A & 71 a A & 50 b A \\
1,0 & 58 b A & 74 a A & 50 b A \\
2,0 & 51 b A & 75 a A & 49 b A \\
\hline 4,0 & 50 b A & 74 a A & 6,0 \\
\hline
\end{tabular}

Médias seguidas de mesma letra maiúscula nas linhas e minúscula, nas colunas, não diferem pelo teste de Tukey ( $5 \%$ de probabilidade). C.V = Coeficiente de Variação 
SILVA.G.C et al. Sementes de feijão-caupi...

De acordo com Silva et al. (2007), o extrato de nim afetou a germinação das sementes e o crescimento das raízes primárias das plântulas de Phaseolus vulgaris. Lobato et al. (2006) testaram sementes de feijão-caupi tratadas com óleo essencial de Piper aduncum L. e verificaram que, à medida em que se aumentaram as concentrações de óleo essencial, há diminuição na percentagem de germinação.

\section{CONCLUSÃO}

O extrato de folhas de nim, reduziu a incidência dos fungos Aspergillus sp e Fusarium sp na cultivares BR 17 e Maranhão, reduziu a incidência de Macrophomina phaseolina somente na cultivar Serrinha e de Phoma sp na cultivar Maranhão.

Houve incremento na germinação da cultivar Maranhão, quando utilizou o extrato de folhas de nim.

\section{REFERÊNCIAS}

1. BRASIL. Ministério da Agricultura. Secretaria Nacional de Defesa Agropecuária. Regras para análise de sementes. Brasília: Ministério da Agricultura, Pecuária e Abastecimento, 2009. 395p.

2. BHUTTA, A.R.; BHATTI, M.H.R.; IFTIKHAR, A. Effect of seed diffusates on fungal population and germination of sunflower seeds. Hélia, v.24, n.34, p.77-81, 2001.

3. CAMPOS, S. Nim - inseticida orgânico. Meio Ambiente/Ecologia. Disponível em: <http:// www.drashirleydecampos.com.br/categorias/53/>. Acesso em: 06 out. 2010.

4. CASA, R.T. Transmissão de fungos em sementes de cereais de inverno e milho: implicações epidemiológicas. In: ZAMBOLIM, L. Sementes: qualidade fitossanitária. Viçosa: UFV, 2005, p. 245-249.

5. FERREIRA, K. C. S.; SANTOS, G. J. C.; SILVA, C. C. Avaliação do tratamento com extrato de nim na redução da incidência de Aspergillus niger associados 'as sementes de embiratanha. Revista Brasileira de Fitopatologia, v. 31 - Suplemento, p. 567,2006.

6. GOVINDACHARI, T.R. et al. Identification of antifungal compounds from the seed oil of Azadirachta indica. Phytoparasitica, v.26, n.2, p.109-116, 1998

7. LOBATO, A. K. S. et al. Germinação de sementes de feijão-caupi tratadas com óleo essencial de Piper aduncum L. In: Reunião Nacional de Feijão-Caupi - RENAC Congresso Nacional de Feijão-Caupi -CONAC 2006. Terezina - PI: Anais do I Congresso Nacional de Feijão - Caupi. EMBRAPA, 2006. p 173.

8. LUCCA FILHO, O.A. Metodologia dos testes de sanidade de sementes. In: SOAVE, J.; WETZEL, M.M.V. da S. (Ed.) Patologia de sementes. Campinas, Fundação Cargill, 1987. p.276-298.

9. MOSSINI, S.A.G.; KEMMELMEIER, C. A árvore Nim (Azadirachta indica. A. Juss.): múltiplos usos. Acta Farmaceutica Bonaerense, v.24, n.1, p.139-148, 2005.

10. PESSOA, M. N. G. et al. Efeito do extrato e do óleo essencial de Ocimum basilicum sobre Colletotrichum gloeosporioides, Macrophomina phaseolina e Lasiodiplodia theobromae. In: Congresso Brasileiro de Fitopatologia 2003. Urlândia - MG: Anais do XXVI Congresso Brasileiro de Fitopatologia. Sociedade Brasileira de Fitopatologia, 2003. p 345

11. PINTO, N. F. J.A. Patologia de sementes de sorgo. Sete Lagoas: EMBRAPA-CNPMS, 1999. (Circular Técnica, 32).

12. SILVA, L. S. et al. Utilização do óleo de nim adicionado via água de irrigação no manejo do mal do panamá. Revista Brasileira de Fitopatologia, v. 31 - Suplemento, p. 223, 2006

13. SILVA, J. P.; CROTTI, A. E. M.; CUNHA, W. Antifeedant and allelopathic activities of the hydroalcoholic extract obtained from Neem (Azadirachta indica) leaves. Brazilian Journal of Pharmacognosy, v. 17, n. 4, p. 529-532, 2007.

14. SOUZA, A. D. et al. R. Efeito de produtos alternativos sobre o crescimento micelial de Penicilium $s p$, Fusarium moniliforme e Fusarium subglutinans in vitro. Summa Phytopathologica, v.32, p. 256, 2006

15. TORRES, S. B.; BRINGEL, J.M.M.. Avaliação da qualidade sanitária e fisiológica de sementes de feijãomacassar. Caatinga, v.18, n.2, p.88-92, 2005.

Recebido em 14/04/2010 Aceito em 17/02/2011 